\title{
Contemporary review in the multi-modality imaging evaluation and management of tricuspid regurgitation
}

\author{
Tom Kai Ming Wang ${ }^{1}$, Shinya Unai ${ }^{2}$, Bo Xu ${ }^{1}$ \\ ${ }^{1}$ Section of Cardiovascular Imaging, Robert and Suzanne Tomsich Department of Cardiovascular Medicine, Sydell and Arnold Miller Family Heart \\ and Vascular Institute, Cleveland Clinic, Cleveland, OH, USA; ${ }^{2}$ Department of Thoracic and Cardiovascular Surgery, Sydell and Arnold Miller \\ Family Heart and Vascular Institute, Cleveland Clinic, Cleveland, OH, USA \\ Contributions: (I) Conception and design: All authors; (II) Administrative support: None; (III) Provision of study materials or patients: None; (IV) \\ Collection and assembly of data: TKM Wang; (V) Data analysis and interpretation: All authors; (VI) Manuscript writing: All authors; (VII) Final \\ approval of manuscript: All authors. \\ Correspondence to: Bo Xu, MB BS (Hons), FRACP, FACC, FASE. Section of Cardiovascular Imaging, Heart and Vascular Institute, Cleveland Clinic, \\ Cleveland, OH, USA. Email: xub@ccf.org.
}

\begin{abstract}
The tricuspid valve has gained interest recently because of the poor outcomes with current treatments and advances in percutaneous valve interventions. A sound understanding of the anatomy and pathologies of the tricuspid valve is critical in its evaluation and management of tricuspid regurgitation (TR). A multi-modality imaging approach with transthoracic echocardiography, transesophageal echocardiography, computed tomography, magnetic resonance imaging all have their individual and collective roles in the evaluation of TR and guidance of surgical and percutaneous procedures. This combined with clinical factors will contribute to defining timing, indications, modality selection and risk stratification for tricuspid valve interventions, which currently remains controversial.
\end{abstract}

Keywords: Tricuspid valve; tricuspid valve insufficiency; echocardiography; X-ray computed tomography; magnetic resonance imaging

Submitted Nov 07, 2019. Accepted for publication Jan 08, 2020.

doi: $10.21037 / \mathrm{cdt} .2020 .01 .06$

View this article at: http://dx.doi.org/10.21037/cdt.2020.01.06

\section{Introduction}

Tricuspid regurgitation (TR) is the main manifestation of tricuspid valve dysfunction, and the presence of at least mild TR is seen in $65-85 \%$ of adults (1). Over the last decade, the tricuspid valve has transformed from being the "forgotten valve" to gaining clinical attention as the one of the next frontiers in structural heart disease. In part this is because of the clinical and adverse prognostic effects of significant TR that is increasingly recognised, in isolation or concurrently with other heart disease $(2,3)$. Equally important is the rapidly evolving landscapes of structural interventions and multimodality imaging which have enabled advances in the assessment and management of TR $(4,5)$. Consequently, even though isolated tricuspid valve surgery is traditionally uncommonly performed, there has been growth in in numbers of this procedure and developments in percutaneous tricuspid interventions (6-9). This article seeks to review the contemporary literature regarding the evaluation and management of TR and its various clinical scenarios with a focus on the utility of multimodality imaging.

\section{Anatomy of the tricuspid valve}

The tricuspid valve serves as the inlet valve of the right ventricle (RV) separating it from the right atrium (RA) (Figure 1). It is generally composed of 3 leaflets labelled anterior, posterior and septal, although anywhere from 2 to 6 leaflets have been reported (10). Via chordae tendinae, 

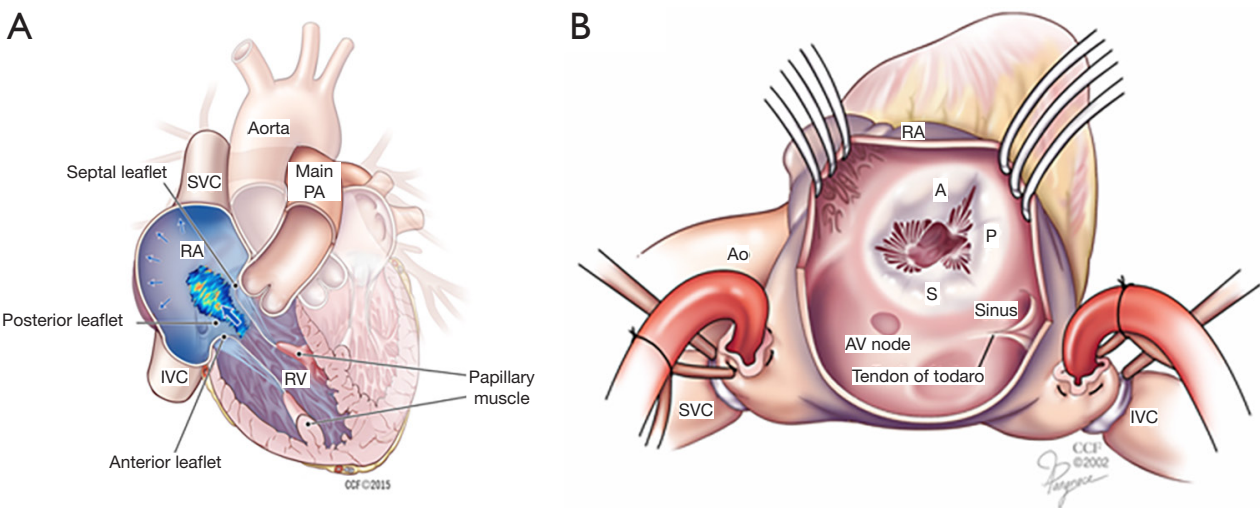

Figure 1 Schematic anatomy of the tricuspid valve apparatus (A) cross-sectional of heart demonstrating tricuspid leaflets, regurgitation, right heart chambers and vasculature; (B) tricuspid valve view from right atrium with leaflet anatomy and important adjacent structures. A, anterior leaflet; S, septal leaflet; P, posterior leaflet. SVC, superior vena cava; IVC, inferior vena cava; PA, pulmonary artery. Note the pulmonary valve is not anatomically adjacent to the tricuspid valve, and the axes of the two valves do not cross vertically as depicted in this illustrative figure.

the leaflets are attached to the papillary muscles in the RV: the anterior papillary muscle is connected to the anterior and posterior leaflets, the posterior papillary muscle is connected to the posterior and septal leaflets and the third and smallest papillary muscle is connected to the anterior and septal leaflets (11). Some of the chordae tendinae of the septal tricuspid leaflet connect directly to the ventricular wall, a feature unique to the tricuspid valve, and not seen for the mitral valve. The tricuspid annulus is a threedimensional triangular and saddle-shaped structure, and elliptical in cross-section, and located more apical than the mitral annulus. Dilation of the annulus in secondary TR results in the annulus losing its saddle shape to form a plane and becoming more circular (12). Figure 1 demonstrates the relevant tricuspid valve anatomy.

For purpose of imaging and interventions of the tricuspid valve, several neighboring structures are worthy of note. The triangle of Koch within the RA is bounded by the septal leaflet of the tricuspid valve, the coronary sinus orifice and the tendon of Todaro, and the apex of this triangle is the atrioventricular node which if damaged can cause heart block (13). The His Bundle heading anteriorly and apically away from the atrioventricular node comes to approximately $5 \mathrm{~mm}$ from the junction of the anterior and septal tricuspid leaflets before going posteriorly beneath the membranous septum (14). The right coronary artery runs an epicardial course coming close to the junction of the anterior and posterior tricuspid leaflets at the atrioventricular groove. The mitral annulus is located to the left of the tricuspid annulus and the aortic valve between and anterior to both.

\section{Etiologies of TR}

\section{Secondary TR}

Table 1 lists the main etiologies for TR. Secondary or functional TR is caused by dilation and/or dysfunction of the right ventricle (RV), resulting in annular dilation and distortion of the tricuspid valve apparatus such as leaflet tethering, rather than diseases affecting the tricuspid valve itself $(15,16)$. The causes can be classified into left heart disease, chronic lung diseases with pulmonary hypertension, atrial fibrillation with annular dilation, primary right heart cardiomyopathy and left-to-right shunts, in descending order of frequency (15). Any left heart pathology that results in increased left atrial and then pulmonary pressures can result in secondary $\mathrm{TR}$, including all types of cardiomyopathy resulting in systolic and/or diastolic dysfunction, left-sided valvular lesions and arrhythmias especially atrial fibrillation. In fact, atrial fibrillation has been found to be associated with right atrial, ventricular and tricuspid annular dilation directly and can lead to right heart failure (17). Chronic lung diseases that can cause pulmonary hypertension with resultant cor pulmonale and secondary TR include chronic obstructive lung disease, asthma, bronchiectasis, interstitial lung disease, obstructive sleep apnoea, pulmonary thromboembolic disease and idiopathic pulmonary hypertension. Less commonly, isolated right ventricular cardiomyopathies, including those 
Table 1 Main etiologies of tricuspid regurgitation

Secondary tricuspid regurgitation
Left heart valve disease
Left heart cardiomyopathy (systolic and/or diastolic dysfunction)
Pulmonary pathology (chronic lung disease, pulmonary
thromboembolism, idiopathic pulmonary hypertension)
Atrial fibrillation with right atrial and annular dilation
Primary right ventricular cardiomyopathy (arrhythmogenic,
ischemic)
Left-to-right shunt (septal defects, anomalous pulmonary
venous return)
Primary tricuspid regurgitation
Rheumatic heart disease
Endocarditis
Myxomatous degeneration/prolapse
Carcinoid syndrome
Radiation
Chest trauma
latrogenic (pacemaker/defibrillator leads, prosthetic valve
degeneration, central line, myocardial biopsy)
hypoplasia, cleft or double orifice)

arrhythmogenic or ischemic in origin, can cause secondary TR. Chronic and substantial left-to-right shunts can also lead to secondary TR. Because of the wide range of common pathologies that can occur in isolation or together that results in functional TR, secondary causes make up the majority of TR encountered in the clinical setting (18). If the right ventricular disease process is adequately treated, the degree of TR often decreases.

\section{Primary TR}

Primary TR is defined by pathology of the tricuspid valve complex leading to regurgitation, and makes up in as little as $10 \%$ of all TR (18). Despite this there are many causes for primary TR including rheumatic heart disease, endocarditis, myxomatous degeneration, carcinoid syndrome, radiation, trauma, iatrogenic and congenital causes. Primary TR can also over time lead to right ventricular dilation and/or dysfunction, which has implications for intervention $(4,5)$. The timing of when TR and RV changes and underlying pathophysiological process would need to be ascertained to determine if the etiology is primary or secondary. To complicate matters, sometimes there may also be mixed primary and secondary causes for the TR. Examples of TR pathologies on echocardiography are shown in Figure 2.

\section{Isolated TR}

Lastly, isolated TR refers to only having clinically significant TR in absence of other valvular disease $(18,19)$. The underlying cause may be primary or secondary. Isolated TR makes up only a small proportion of TR because the majority of TR etiologies affect multiple valves $(9,20)$. For example, rheumatic heart disease and endocarditis frequently affects mitral and/or aortic valves and carcinoid syndrome affects the pulmonary valve. Other etiologies like myxomatous degeneration, trauma, iatrogenic and congenital may only affect the tricuspid valve. These have important implications for both imaging and intervention.

\section{Imaging assessment of TR severity}

\section{Echocardiography}

Table 2 summarises the key features of cardiac imaging modalities for assessing TR. Transthoracic echocardiography (TTE) is the first-line imaging modality to assess valvular heart disease including TR $(4,5,21)$. The views for assessing TR and RV include the parasternal long axis RV inflow view, parasternal short axis view focusing on tricuspid valve, apical 4-chamber including the RV focused view, subcostal 4-chamber view and the subcostal long axis inferior vena cava and hepatic vein view (22). The morphology of the tricuspid valve needs careful examination to provide hints on the underlying etiology and location of TR. Many echocardiographic measurements are used to assess TR severity including the semiquantitative measurements of colour flow jet area, vena contracta width $(>7 \mathrm{~mm}$ is severe), proximal isovelocity surface area radius ( $>9 \mathrm{~mm}$ at $40 \mathrm{~cm} / \mathrm{s}$ Nyquist is severe), hepatic vein flow (systolic reversal is severe) and tricuspid inflow (E-wave $>1 \mathrm{~m} / \mathrm{s}$ dominant), as well as quantitative measurements of effective regurgitant orifice area $\left(>0.4 \mathrm{~cm}^{2}\right)$ and regurgitant volume $(>45 \mathrm{~mL})$, each with their unique utility and limitations (21). TR severity are graded as mild, moderate or severe following algorithms in guidelines, with different thresholds depending on parameter.

The RV must also be assessed in evaluating TR severity, 

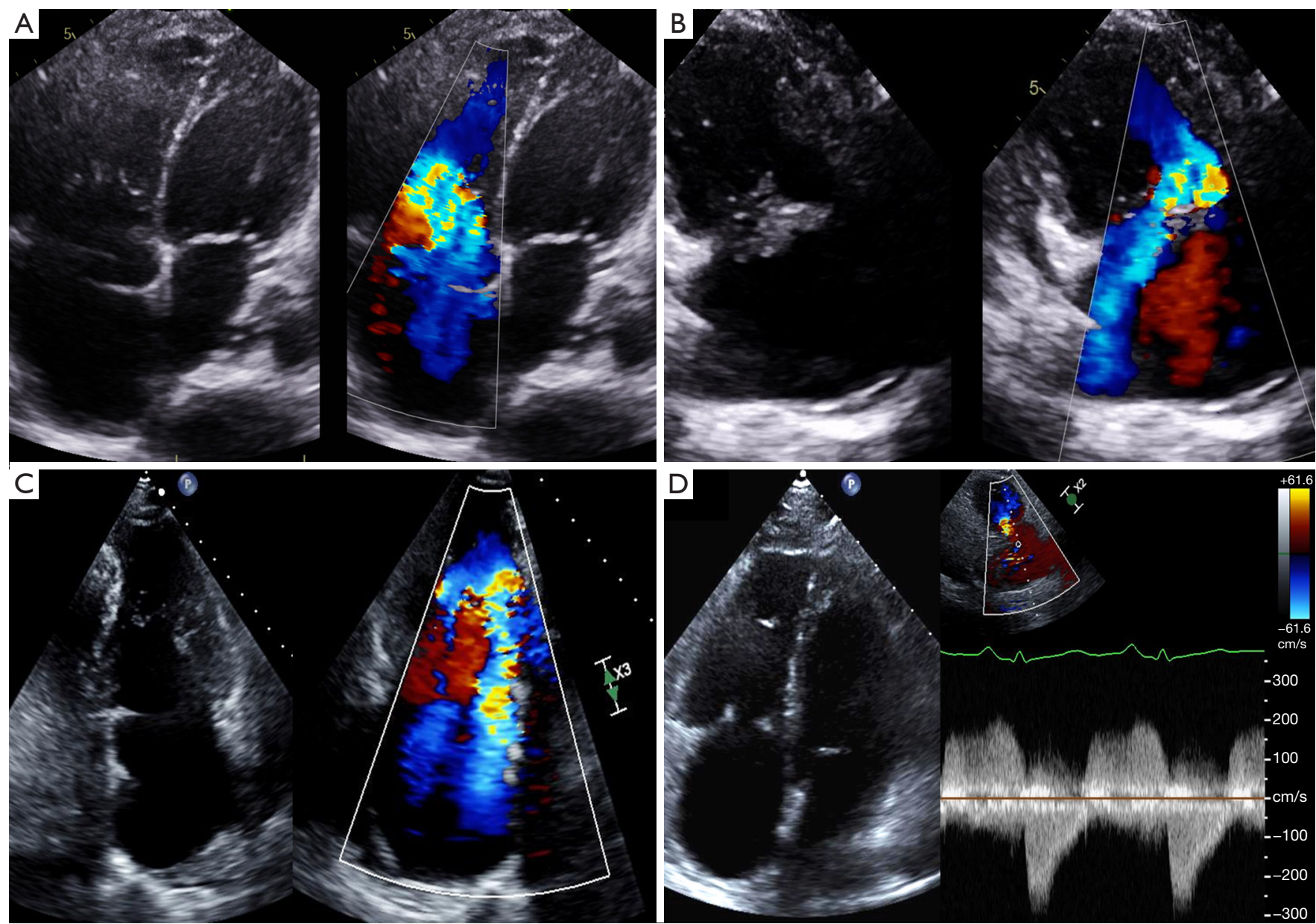

Figure 2 Varying etiologies of tricuspid regurgitation on echocardiography (A) functional tricuspid regurgitation on transthoracic echocardiography apical four-chamber view with color Doppler, (B) native tricuspid valve endocarditis associated with significant tricuspid regurgitation on transthoracic echocardiography parasternal tricuspid inflow view with color Doppler (C) Ebstein anomaly associated tricuspid regurgitation demonstrated on transthoracic echocardiography parasternal tricuspid inflow view with color Doppler and (D) Carcinoid syndrome with mixed tricuspid regurgitation and stenosis on right ventricular focused apical 4-chamber view and continuous wave Doppler.

because RV dilation and/or systolic dysfunction are also potential indications of tricuspid valve surgery $(4,5,21)$. The RV is commonly dilated when there is significant TR, with septal flattening towards and compression towards the left ventricle in diastole in setting of RV volume overload, and throughout the cardiac cycle particularly systole in RV pressure overload in presence of pulmonary hypertension (21). There are several parameters with defined normal ranges to assess RV systolic function, including annular systolic excursion, S-wave Doppler (pulsed or colour), fractional area change, RV free wall strain, tricuspid inflow, and $3 \mathrm{D}$ ejection fraction calculation (23).
Notably, in absence of accurate quantitative measures, dilation of the RV annular dimension on apical 4-chamber view with tricuspid annulus $\geq 40 \mathrm{~mm}$ or $>21 \mathrm{~mm} / \mathrm{m}^{2}$ is a surrogate measure of TR severity and supports surgery (4).

Transesophageal echocardiogram (TEE) is frequently utilised in the setting when windows are suboptimal for TR assessment on TTE, despite the anterior location of the tricuspid valve. Important tricuspid valve views on TEE include the mid-esophageal four chamber, right ventricular inflow/outflow, bicaval tricuspid and reverse four chamber views, 3D reconstruction of the tricuspid valve, and transgastric RV inflow +/- outflow views $(13,24)$. 
Table 2 Summary of cardiac imaging utility for evaluating TR and guiding interventions

Transthoracic echocardiography

Strengths: portable, accessible, low-cost, safe

Weaknesses: lowest spatial resolution, extracardiac structures/ right heart

TR and other valves severity and etiology (qualitative and quantitative)

Chamber size and systolic function

Pulmonary pressure estimate

2D, Doppler and 3D assessment

Periprocedural complications and serial follow-up

Transesophageal echocardiography

Strengths: superior to transthoracic for many structures

Weaknesses: invasive, require sedation/anesthesia affects loading,

TR and other valves severity and etiology (qualitative and quantitative)

Chamber size and systolic function

Pulmonary pressure estimate

2D, Doppler and 3D assessment

Intraoperative guidance of percutaneous interventions

Computed tomography

Strengths: spatial resolution, multiplanar reconstruction, extracardiac structure assessment

Weaknesses: radiation, contrast, valve assessment

Anatomy/relationships with neighboring structures intracardiac and extracardiac

Planning transcatheter procedures

Magnetic resonance imaging

Strengths: spatial resolution, multiplanar reconstruction, tissue characterization, extracardiac

Weaknesses: claustrophobia, cardiac devices, contrast, cost

TR severity quantitative

Chamber size and systolic function (gold standard) and tissue characterization

Anatomy/relationships with neighboring structures intracardiac and extracardiac
The same parameters for TTE can be measured on TEE, recognising their limitations and the need to minimise the angle between the colour jet and Doppler interrogation. TEE, including 3D is strongly recommended to assessing TR with indeterminate severity, aetiology and/or prior to intervention. 3D TEE can give further detail to tricuspid valve anatomy, as well as the severity, aetiology, origin and direction of TR, especially with the help of multiplanar reconstruction and real-time images. The loading conditions may change the TR severity when sedatives and/ or anesthesia are administered causing under-estimation and need to be accounted for. TEE can also visualise other structures nearby better than TTE such as the interatrial septum, SVC and IVC.

\section{Computed tomography (CT)}

CT has several advantages for cardiac imaging in terms of rapid acquisition, high spatial resolution, availability and ability for multi-planar reconstruction (25). It can determine dimensions and function of relevant structures including RA, RV, tricuspid annulus as well as neighbouring vasculature and anomalies such as both vena cavae, pulmonary vessels, coronary vessels and aorta with good correlation to magnetic resonance imaging (MRI) (26). CT coronary angiography if demonstrating no significant coronary disease can avoid the need for conventional catheter angiography required for intervention workup. Acquisition of cardiac cycle to quantify TR can be performed, although MRI is usually preferred to minimise radiation, and iodinated contrast is another contraindication in renal failure patients. However in patients with pacemakers and defibrillators which are not MRI compatible, CT is a useful alternative although related artefacts will need to be interpreted as well.

Tricuspid valve imaging on CT may not be straightforward, given the inferior temporal resolution to echocardiography for mobile structures, and conventional studies for the left heart leave the right heart filled with contrast, making interrogation of the tricuspid valve structure tricky (27). However, dedicated tricuspid valve imaging and contrast protocols using multi-detector CT with electrocardiogram gating, anatomical evaluation of 
the tricuspid valve can be performed with optimal contrast timing. Heart rate control remains important to minimise motion artefact, and irregular rhythms such as atrial fibrillation may render imaging difficult (28). CT is also useful in planning percutaneous interventions as will be described later.

\section{Cardiac MRI}

MRI offers complimentary information for assessment and surveillance of TR, especially if echocardiogram views are suboptimal, when TR severity is indeterminate, and to assess causes for underlying cardiomyopathy (4). It has superior spatial resolution to TTE with adequate temporal resolution for accurate cardiac chamber and valve assessment and radiation-free unlike CT. MRI is the reference standard for RV volume, which tends to be under-estimated by echocardiography, function and mass $(29,30)$. Biventricular dimensions and function are usually assessed on MRI by short-axis stack of the steady state free precession cine imaging which has good myocardial to blood-pool contrast (27). Dedicated RV views can be acquired including conventional 4-chamber view and RV 2-chamber, 3-chamber and outflow tract views. Regional wall motion abnormalities and even strain of the RV can be evaluated although the utility for TR is currently uncertain.

MRI is useful in many other ways. It can assess TR severity, for example calculating the difference between RV stroke volume and pulmonary or aortic flow, the latter with phase contrast velocity encoding imaging. Another parameter is the cross-sectional TR jet area which increases and signal intensity decreases in shortaxis steady-state free precession images as TR severity increases (31). The tricuspid valve anatomy can sometimes be inspected on MRI including prolapse, tenting and thickening. The unique ability for tissue characterization such as late gadolinium enhancement and T1-imaging well established for left ventricular cardiomyopathy is under research for RV pathology which is often thin-walled and TR (30). MRI can like CT also depict vasculature anatomy and assess congenital heart disease and anomalies. Its limitations include longer acquisition time, cardiac devices, claustrophobia, artefacts, contrast for certain protocols and still susceptible to motion artefacts including breathing.

\section{Indications for TR interventions}

\section{Guidelines}

Valvular heart disease guidelines have evolved significantly in recent years mainly because of the advances in the management of aortic stenosis and mitral regurgitation $(4,5,32)$. This has not yet applied to the management of TR, which can be seen by the 2017 American College of Cardiology (ACC)/American Heart Association (AHA) update of the 2014 guidelines not having a section on TR (32). This is a result of both the lack of randomised trials or approved percutaneous devices for TR. Although the latter continues to be in development, until then the evidence is limited and recommendations have remained similar for the indication of TR intervention in the last few iterations of the American and European Society of Cardiology (ESC) guidelines as seen in Table $3(4,5,32)$.

The ACC/AHA guidelines 2014 for valvular heart disease recommend intervention for the tricuspid valve at the time of left-sided valve surgery if there was severe TR or progressive mild or moderate functional TR. Isolated TR surgery is recommended in patients with severe TR with either symptoms or progressive RV dysfunction (5). The corresponding ESC guidelines in 2017 also suggest tricuspid valve intervention with left heart valve surgery when TR is severe, or mild or moderate with right heart failure or tricuspid annular dilatation $(4,14)$. For isolated TR surgery, patients need to have severe TR with either symptoms or progressive RV dilation and/or dysfunction, however particularly in those with secondary, also severe LV dysfunction, RV dysfunction or pulmonary hypertension would push towards conservative management because of excessive surgical risk.

\section{Guidelines evidence and limitations and recent data}

These recommendations are reported in the class I to IIb range, however the level of evidence is only $\mathrm{C}$ for all, which means that they are based on expert opinion and observational studies $(4,5)$. There are some recent data such as a study-level meta-analysis from 2017 of 15 observational studies and 2,840 patients, whereby for concomitant TR surgery to left-sided valve surgery had significantly lower cardiovascular mortality and trend towards lower 
Table 3 Guideline recommendations of tricuspid surgery indications

ACC/AHA 2014
Severe primary TR + symptoms (Ila, C)
Severe TR + left-sided valve surgery (I, C)
Asymptomatic severe TR + progressive RV dysfunction (I, C for
secondary, Ilb, C for primary)
Mild/moderate progressive functional TR + left-sided valve
surgery + TA dilation (Ila, B) or PHTN (Ilb, C)
Severe TR with symptoms and previous left-sided valve surgery,
without severe PHTN or RV dysfunction (Ilb, C)
ESC 2017
Left-sided valve surgery + severe TR (I, C)
Left-sided valve surgery + mild/moderate TR +
TA dilation (Ila, C) or recent signs of right heat failure (Illb, C)
Severe primary TR + symptoms (I, C)
Severe secondary TR + severe RV or LV dysfunction
or severe PHTN + symptoms (Ila, C)
Severe secondary TR + severe RV or LV dysfunction
or severe PHTN + progressive RV dilation/dysfunction (Ila, C)
ACC/AHA, American College of Cardiology/American Heart
Association; ESC, European Society of Cardiology; TR, tricuspid
regurgitation; (), level of evidence (class I = recommended,
Ila = should be considered, Ilb = may be considered, III = not
clinical trials or meta-analyses, B = single randomized clinical
and/or small studies, retrospective studies or registries), RV,
pulmonary hypertension,

all-cause mortality, possibly related to decreased grade and progression of TR during follow-up (33). Another randomized trial found tricuspid annuloplasty concomitant at time of mitral valve surgery reduced not only TR longterm but also pulmonary pressures (34). The data are lacking for establishing the optimal timing for surgery, and if isolated tricuspid valve surgery has superior survival to medical management, in fact a recent study reported no benefits with a surgical approach (35).

There are no guidelines currently for supporting the use or establishing indications of percutaneous tricuspid interventions $(4,5)$. Its role will likely begin in those as a concomitant procedure to percutaneous mitral or aortic valve intervention, and high-risk or inoperative patients with TR. In fact, the majority of these patients may have isolated TR patients. Certainly further developments of this technology and research are necessary and upcoming.

\section{Management options for TR}

\section{Medical therapy}

Medical management remains the first line management of TR (5). Right heart failure symptoms predominate in peripheral edema and ascites however in secondary causes pulmonary congestion is also often present as the reason for hospitalisation (36). Diuretic therapy are the mainstay medical option, including loop diuretics and aldosterone antagonists, not infrequently at high doses and/or in combinations $(5,17)$. Following this, therapies directed at the underlying etiology of TR whether primary or secondary is important. For primary TR this may include antibiotics for endocarditis, anti-inflammatories and antibiotics for rheumatic heart disease and somatostatin analogues and other therapies for carcinoid syndrome. Left heart disease, chronic lung disease and in some scenarios pulmonary hypertension itself needs adequate medical therapy which may lead to regression of secondary TR $(37,38)$.

\section{Surgical techniques}

Surgery remains the gold standard and up until recently the only viable intervention for TR $(4,5)$. Indications are as described although evidence is limited to observational studies and expert opinion. Isolated tricuspid valve surgery makes up only $15-20 \%$ of all tricuspid valve surgery, the majority of which are concurrent with mitral, aortic and/ or coronary surgery (9). Operating on the tricuspid valve however puts the surgery at moderate or high risk despite the growth in procedures performed $(6,7)$. Operative mortality rates have continued to be reported at around $10 \%$ for both isolated and concomitant tricuspid surgeries and both primary and secondary TR in many large contemporary registries $(7,9,39,40)$, compared to only $2-3 \%$ for mitral and aortic valve surgeries (20). Reasons for the much higher risk for tricuspid surgery may are likely related to high prevalence of right ventricular dysfunction, chronic lung disease and pulmonary hypertension, liver dysfunction, previous cardiac surgery and other co-morbidities, as well as delayed referral, and these issues need to be dealt with to improve surgical outcomes $(4,6)$.

The two classes of surgical techniques are valve repair and replacement. Repair techniques are based on annuloplasty to reduce the area and improve coaptation of leaflets. 
This can be accomplished with continuous sutures such as De Vega annuloplasty, avoiding prosthetic material (41). Another strategy is implantation of an incomplete prosthetic ring sutured to the annulus, which may be rigid or flexible, and careful sizing is required. Leaflet augmentation and suture bicuspidization of the tricuspid valve are other techniques. Ring annuloplasty appears to reduce residual TR and possibly improve clinical outcomes compared to suture and flexible band annuloplasty so is usually preferred $(42,43)$. The main decision to make for valve replacement is between mechanical and bioprosthetic valves. A metaanalysis of 22 observational studies reported no difference in survival, prosthetic valve failure or re-operation, however thrombosis rates were higher for mechanical valves (44). This has been reflected in large registries where although both prosthesis make up about half of replacement surgeries, there is increased use of bioprosthetic valves and decreased use of mechanical valves over time (9). Most studies and a meta-analysis report higher rates of operative mortality, and pacemaker implantation, for replacement compared to repair including for secondary TR $(7,9,39,45)$, while others found no difference (40). As a result guidelines recommend tricuspid repair to be performed where feasible, however sometimes because of markedly damaged and/ or distorted tricuspid valve apparatus, valve replacement cannot be avoided $(4,5)$.

\section{Percutaneous intervention}

With the paradigm shift towards minimally invasive and percutaneous procedures for structural heart disease over the last decade, development, research and early clinical use of percutaneous tricuspid valve interventions are underway $(4,14)$. Devices used are grouped into categories of coaptation, annuloplasty and prosthetic valve or heterotopic caval valve implantation (13). Coaptation devices include the Mitra-clip (Abbot, Santa Clara, CA, United States) used in off-label fashion as edge-to-edge repair of the tricuspid valve, and foam-filled polymer balloon placed distal to the valve towards the RV to provide surface for improved coaptation (46). Annuloplasty systems aim to improve the distorted geometry of the annulus in functional TR, with strategies including pledgeted sutures to cinch commissures to bicuspidize the valve, adjustable band placement and annuloplasty ring implantation (47). Prosthetic valve implantation has mainly been used in those with preexisting bioprosthetic valve as a valve-in-valve technique, originally in young congenital heart disease patients but now expanding (48). The heterotopic caval valve is placed in the superior and/or inferior vena cava to reduce regurgitation.

Percutaneous interventions are currently performed in patients with high surgical risk, with the vast majority in secondary TR, and a high prevalence of previous cardiac surgery (49). Despite this, early outcomes have been reportedly satisfactory, with an international registry reporting operative mortality of $3.6 \%$, lower in those with procedural success which occurred in the majority (8). Another recent propensity-matched observational study found percutaneous tricuspid repair to reduce clinical events including all-cause mortality and heart failure hospitalizations at 1-year (26). Further research is mandatory and ongoing to further refine tricuspid device technology, provide larger scale data on peri-operative outcomes, long-term survival and durability, as well as comparisons with surgical and medical therapies, before wider use in clinical applications.

\section{Peri-procedural imaging for TR interventions}

\section{Echocardiography}

Important features of how cardiac imaging modalities can be used to guide TR interventions are listed in Table 2. As described earlier echocardiography remains first line and central to the assessment of TR severity, etiology and overall tricuspid valve anatomy. TEE is helpful intraoperatively to assessing TR, other valves and cardiac function, including in scenario of general anaesthetic loading conditions. It provides live continuous imaging including to guide catheter positioning and reduce fluoroscopy. The key transesophageal and transgastric TEE views were described earlier, and furthermore the use of three-dimensional TEE with multi-planar reconstruction enables delineation of device position relative to the pathology being treated real time, as illustrated in Figure $3(13,24)$. Intracardiac echocardiography via vascular sheath and single-use probe intraoperatively may further provide complementary information.

After the intervention is performed, echocardiography remain first line both intra- or post-operatively in the assessment of residual TR and cardiac function (50). Where possible, measures used in pre-operative assessment of TR and $\mathrm{RV}$ in accordance to guidelines. 3D echocardiography with TEE are particularly useful at visualising residual TR with sensitivity and specificity in excess of $80 \%$ (51). There 

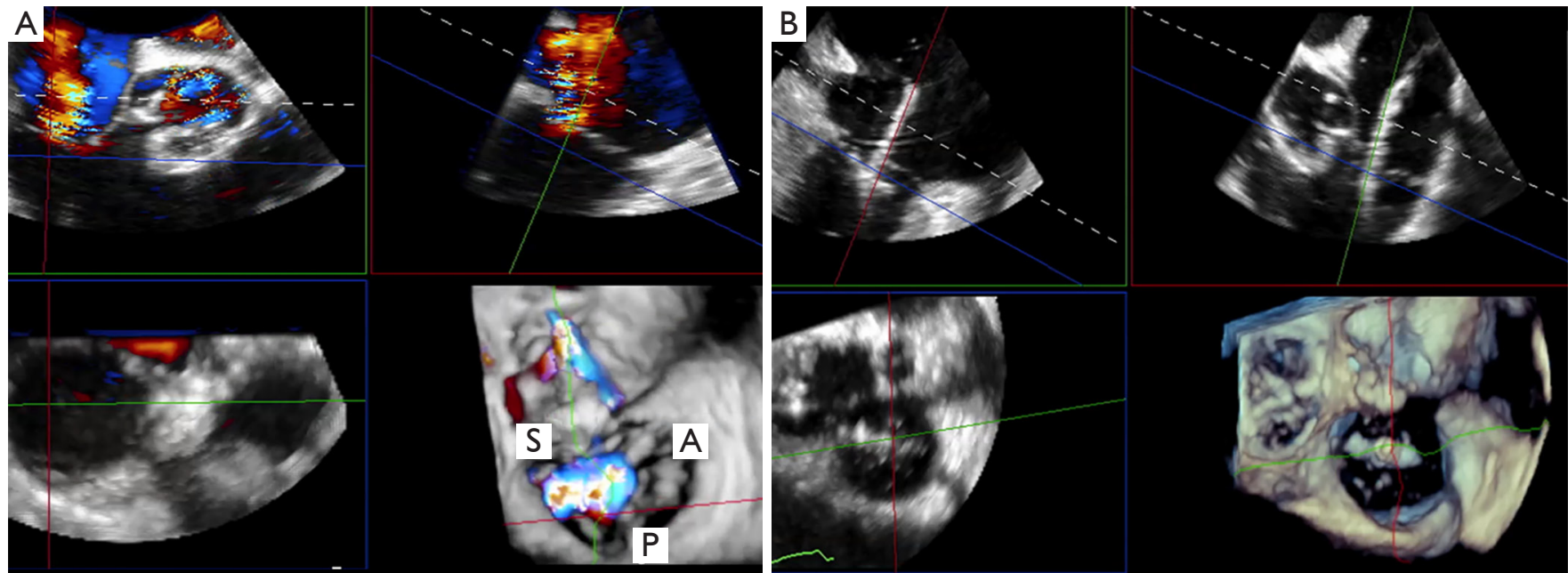

Figure 3 Three-dimensional transesophageal echocardiography with multiplanar reconstruction to (A) evaluate location of tricuspid regurgitation (S: septal leaflet, A: anterior leaflet and P: posterior leaflet of tricuspid valve), and (B) to guide percutaneous tricuspid repair with edge-to-edge closure.

are a number of challenges with presence of a variety of tricuspid prosthesis or devices, and lack of studies validating these measures which require further research and experience as newer devices are in use.

\section{Cardiac CT}

CT is a valuable tool for all percutaneous interventions including for TR $(4,52)$. It has the ability to characterise the anatomy and spatial relationships between the tricuspid valve and neighbouring structures within and outside the heart. Many patients have had previous cardiac surgery, and CT scan can assess the any resultant distortion in cardiac anatomy, and the proximity of the sternum to the cardiac chambers, coronary vessels and grafts where applicable. CT can assess the vasculature for anomalies, obstruction and peripheral vessels for suitability of access and route to the heart (25). It can analyse the angles, lengths and relationships of the superior and inferior vena cava entering the right atrium and distance from the first hepatic vein to plan for caval valve implantation. The right coronary artery course and proximity to the tricuspid annulus can be depicted to assess suitability of different annuloplasty systems. The tricuspid annulus dimensions and distance to the RV apex helps plan for coaptation devices.

\section{Cardiac MRI}

MRI has important roles in assessing TR severity and is the most accurate modality for assessing RV dimensions and function which has implications for indication and prognosis (53). It is also useful to characterise abnormal cardiac chamber and vasculature anatomy such as in congenital heart disease, and this information is important too for tricuspid interventions. Etiology and leaflet anatomy assessment continues to be inferior to echocardiography and CT, and it has no current role intraoperatively. MRI however remains a useful alternative to CT with there are contraindications like renal failure to avoid contrast and lack of radiation. MRI also has a role in assessing residual TR after tricuspid valve interventions using similar techniques to pre-operative TR severity assessment described earlier using the difference of RV stroke volume and flow across the pulmonary and/or aortic valve (50).

\section{Fluoroscopy}

Fluoroscopy remains invaluable during percutaneous valve interventions, performed in either the cardiac catheterization laboratory or hybrid operating theaters. A set of standard fluoroscopic views have been defined to better assess right heart structures: the 1-chamber short-axis view (across tricuspid valve) at left anterior oblique with caudal projection, 2-chamber view (right atrium and ventricle added) at right anterior oblique with caudal projection, 3-chamber view (include right ventricular outflow tract into main pulmonary artery and valve), at right anterior oblique 
with cranial projection and 4-chamber view (four cardiac chambers) at left anterior oblique and cranial projection (54). The bicaval and right ventricular outflow tract to pulmonary artery are other useful views. Fluoroscopy views can also be reconstructed on CT with overlay onto fluoroscopy images. These help with identifying catheter position within the right heart relative to other landmarks to assist tricuspid interventions.

\section{Risk prediction for TR interventions}

\section{Mortality and morbidities}

Risk models are widely used and recommended by guidelines in the pre-operative assessment of cardiac surgery for stratification and decision-making $(4,5)$. The two most commonly used risk scores currently are the Society of Thoracic Surgeon's (STS) Score and EuroSCORE II (20,55). Both scores estimate operative mortality in-hospital and/or within 30-day, while the STS Score uniquely has separate models by type of surgery and morbidity outcomes. Despite the high risk nature of tricuspid valve surgery, risk models have been rarely studied in this context. Only 5\% of the EuroSCORE II developmental cohort had tricuspid valve surgery (55) and the STS family of models were not designed to be applicable to tricuspid valve surgery until the recently published STS tricuspid score (56). This score did not have separate models for tricuspid repair or replacement like the mitral valve STS scores. Furthermore, none of these scores have been externally validated in tricuspid valve surgery. Interesting the modified model for endstage liver disease score has been found to have moderate discrimination for composite adverse events (57).

Most of the parameters of existing cardiac surgery scores would likely apply to tricuspid valve surgery, but there are notable classes of risk factors missing. The first class is imaging, where only the left ventricular ejection fraction is part of existing risk models (55). Other potential parameters include right ventricular function and size, despite being contributors to surgical indications, echocardiographic measures, and cardiac MRI markers like late gadolinium enhancement and T1 mapping $(58,59)$. The second group of variables is biomarkers, where only renal function is considered, but others including cardiac biomarkers such as B-type natriuretic peptide and troponins, liver function like albumin, and even genomics and metabolomics may play a role (60). Finally factors such as frailty may also play a role in all cardiac interventions particularly in older and/or co-morbid patients (61). Reasons these are not incorporated in risk models are most likely because they are not routinely collected rather than not being significant, whether as a result of the lack of standardised techniques for measurements, or the extra time and costs involved to do so. Further research is required towards incorporation of these classes and others of parameters, with consideration of novel techniques involving machine learning and data science, to refine the accuracy of existing risk models and improve clinical applicability.

\section{Recurrent TR}

Beyond predicting mortality and morbidities after tricuspid valve surgery, some studies have investigated the predictors of TR recurrence $(62,63)$. This is an important surrogate measure because of being associated with worse clinical outcomes and the need for re-operation (64). Important predictors of significant TR recurrence early and/or late include imaging parameters such as valve tethering height and area, TR severity, annular diameter and left ventricular ejection fraction; and clinical factors such as atrial fibrillation, presence of pacemaker lead and renal impairment $(62,63,65)$. Thorough assessment for these factors pre-operatively is important as the presence of one or multiple predictors would caution the clinicians involved to pay extra attention in the surgical and peri-operative care of TR patients.

\section{Conclusions}

Tricuspid valve and TR have gained significant interests as the next frontier in valvular heart disease and structural interventions. Although TTE is the first-line workhorse in TR assessment, TEE, CT and MRI all play important roles in the pre-operative assessment and guidance of interventions. Tricuspid valve surgery remains a high risk operation even in contemporary registries, where there will likely be an important role for transcatheter tricuspid interventions. Future directions lie in development of percutaneous therapies, larger registries and randomised trials comparing surgical, percutaneous and medical therapies and to better define indications and timing for TR intervention, and further experiences and advances in multi-modality imaging to contribute to decision-making, procedure guidance and risk prognostication. 


\section{Acknowledgments}

Funding: TKMW is supported by the National Heart Foundation of New Zealands Overseas Clinical and Research Fellowship - grant number 1775.

\section{Footnote}

Provenance and Peer Review: This article was commissioned by the editorial office, Cardiovascular Diagnosis and Therapy for the series "Heart Valve Disease". The article has undergone external peer review.

Conflicts of Interest: All authors have completed the ICMJE uniform disclosure form (available at http://dx.doi. org/10.21037/cdt.2020.01.06). The series "Heart Valve Disease" was commissioned by the editorial office without any funding or sponsorship. Xu B served as the unpaid Guest Editor of the series. Dr. TKMW reports grants from National Heart Foundation of New Zealand, during the conduct of the study. The authors have no other conflicts of interest to declare.

Ethical Statement: The authors are accountable for all aspects of the work in ensuring that questions related to the accuracy or integrity of any part of the work are appropriately investigated and resolved.

Open Access Statement: This is an Open Access article distributed in accordance with the Creative Commons Attribution-NonCommercial-NoDerivs 4.0 International License (CC BY-NC-ND 4.0), which permits the noncommercial replication and distribution of the article with the strict proviso that no changes or edits are made and the original work is properly cited (including links to both the formal publication through the relevant DOI and the license). See: https://creativecommons.org/licenses/by-nc-nd/4.0/.

\section{References}

1. Singh JP, Evans JC, Levy D, et al. Prevalence and clinical determinants of mitral, tricuspid, and aortic regurgitation (the Framingham Heart Study). Am J Cardiol 1999;83:897-902.

2. Nath J, Foster E, Heidenreich PA. Impact of tricuspid regurgitation on long-term survival. J Am Coll Cardiol 2004;43:405-9.

3. Sadeghpour A, Hassanzadeh M, Kyavar M, et al. Impact of severe tricuspid regurgitation on long term survival. Res Cardiovasc Med 2013;2:121-6.

4. Baumgartner H, Falk V, Bax JJ, et al. 2017 ESC/EACTS Guidelines for the management of valvular heart disease. Eur Heart J 2017;38:2739-91.

5. Nishimura RA, Otto CM, Bonow RO, et al. 2014 AHA/ ACC guideline for the management of patients with valvular heart disease: executive summary: a report of the American College of Cardiology/American Heart Association Task Force on Practice Guidelines. J Am Coll Cardiol 2014;63:2438-88.

6. Alqahtani F, Berzingi CO, Aljohani S, et al. Contemporary Trends in the Use and Outcomes of Surgical Treatment of Tricuspid Regurgitation. J Am Heart Assoc 2017;6. doi: 10.1161/JAHA.117.007597.

7. Zack CJ, Fender EA, Chandrashekar P, et al. National Trends and Outcomes in Isolated Tricuspid Valve Surgery. J Am Coll Cardiol 2017;70:2953-60.

8. Taramasso M, Alessandrini H, Latib A, et al. Outcomes After Current Transcatheter Tricuspid Valve Intervention: Mid-Term Results From the International TriValve Registry. JACC Cardiovasc Interv 2019;12:155-65.

9. Vassileva CM, Shabosky J, Boley T, et al. Tricuspid valve surgery: the past 10 years from the Nationwide Inpatient Sample (NIS) database. J Thorac Cardiovasc Surg 2012;143:1043-9.

10. Wafae N, Hayashi H, Gerola LR, et al. Anatomical study of the human tricuspid valve. Surg Radiol Anat 1990;12:37-41.

11. Ginns J, Ammash N, Bernier PL. The tricuspid valve in adult congenital heart disease. Heart Fail Clin 2014;10:131-53.

12. Mahmood F, Kim H, Chaudary B, et al. Tricuspid annular geometry: a three-dimensional transesophageal echocardiographic study. J Cardiothorac Vasc Anesth 2013;27:639-46.

13. Kim SM, Singh HS, Nati J, et al. Multi-Modality Imaging in the Evaluation and Treatment of Tricuspid Regurgitation. Curr Treat Options Cardiovasc Med 2018;20:77.

14. Antunes MJ, Rodriguez-Palomares J, Prendergast B, et al. Management of tricuspid valve regurgitation: Position statement of the European Society of Cardiology Working Groups of Cardiovascular Surgery and Valvular Heart Disease. Eur J Cardiothorac Surg 2017;52:1022-30.

15. Tornos Mas P, Rodriguez-Palomares JF, Antunes MJ. Secondary tricuspid valve regurgitation: a forgotten entity. Heart 2015;101:1840-8. 
16. Sagie A, Schwammenthal E, Padial LR, et al. Determinants of functional tricuspid regurgitation in incomplete tricuspid valve closure: Doppler color flow study of 109 patients. J Am Coll Cardiol 1994;24:446-53.

17. Najib MQ, Vinales KL, Vittala SS, et al. Predictors for the development of severe tricuspid regurgitation with anatomically normal valve in patients with atrial fibrillation. Echocardiography 2012;29:140-6.

18. Mutlak D, Lessick J, Reisner SA, et al. Echocardiographybased spectrum of severe tricuspid regurgitation: the frequency of apparently idiopathic tricuspid regurgitation. J Am Soc Echocardiogr 2007;20:405-8.

19. Fender EA, Zack CJ, Nishimura RA. Isolated tricuspid regurgitation: outcomes and therapeutic interventions. Heart 2018;104:798-806.

20. O'Brien SM, Feng L, He X, et al. The Society of Thoracic Surgeons 2018 Adult Cardiac Surgery Risk Models: Part 2-Statistical Methods and Results. Ann Thorac Surg 2018;105:1419-28.

21. Zoghbi WA, Adams D, Bonow RO, et al. Recommendations for Noninvasive Evaluation of Native Valvular Regurgitation: A Report from the American Society of Echocardiography Developed in Collaboration with the Society for Cardiovascular Magnetic Resonance. J Am Soc Echocardiogr 2017;30:303-71.

22. Mitchell C, Rahko PS, Blauwet LA, et al. Guidelines for Performing a Comprehensive Transthoracic Echocardiographic Examination in Adults: Recommendations from the American Society of Echocardiography. J Am Soc Echocardiogr 2019;32:1-64.

23. Lang RM, Badano LP, Mor-Avi V, et al. Recommendations for cardiac chamber quantification by echocardiography in adults: an update from the American Society of Echocardiography and the European Association of Cardiovascular Imaging. J Am Soc Echocardiogr 2015;28:1-39.e14.

24. Hahn RT, Abraham T, Adams MS, et al. Guidelines for performing a comprehensive transesophageal echocardiographic examination: recommendations from the American Society of Echocardiography and the Society of Cardiovascular Anesthesiologists. J Am Soc Echocardiogr 2013;26:921-64.

25. van Rosendael PJ, Kamperidis V, Kong WK, et al. Computed tomography for planning transcatheter tricuspid valve therapy. Eur Heart J 2017;38:665-74.

26. Taramasso M, Benfari G, van der Bijl P, et al. Transcatheter Versus Medical Treatment of Patients With Symptomatic Severe Tricuspid Regurgitation. J Am Coll Cardiol
2019;74:2998-3008.

27. Khalique OK, Cavalcante JL, Shah D, et al. Multimodality Imaging of the Tricuspid Valve and Right Heart Anatomy. JACC Cardiovasc Imaging 2019;12:516-31.

28. Hinzpeter R, Eberhard M, Burghard P, et al. Computed tomography in patients with tricuspid regurgitation prior to transcatheter valve repair: dynamic analysis of the annulus with an individually tailored contrast media protocol. EuroIntervention 2017;12:e1828-36.

29. Tamborini G, Piazzese C, Lang RM, et al. Feasibility and Accuracy of Automated Software for Transthoracic Three-Dimensional Left Ventricular Volume and Function Analysis: Comparisons with Two-Dimensional Echocardiography, Three-Dimensional Transthoracic Manual Method, and Cardiac Magnetic Resonance Imaging. J Am Soc Echocardiogr 2017;30:1049-58.

30. Kramer CM, Barkhausen J, Flamm SD, et al. Standardized cardiovascular magnetic resonance (CMR) protocols 2013 update. J Cardiovasc Magn Reson 2013;15:91.

31. Medvedofsky D, Leon Jimenez J, Addetia K, et al. Multiparametric quantification of tricuspid regurgitation using cardiovascular magnetic resonance: A comparison to echocardiography. Eur J Radiol 2017;86:213-20.

32. Nishimura RA, Otto CM, Bonow RO, et al. 2017 AHA/ ACC Focused Update of the 2014 AHA/ACC Guideline for the Management of Patients With Valvular Heart Disease: A Report of the American College of Cardiology/ American Heart Association Task Force on Clinical Practice Guidelines. J Am Coll Cardiol 2017;70:252-89.

33. Pagnesi M, Montalto C, Mangieri A, et al. Tricuspid annuloplasty versus a conservative approach in patients with functional tricuspid regurgitation undergoing leftsided heart valve surgery: A study-level meta-analysis. Int J Cardiol 2017;240:138-44.

34. Pettinari M, De Kerchove L, Lazam S, et al. Mid-term results of a randomized trial of tricuspid annuloplasty for less-than-severe functional tricuspid regurgitation at the time of mitral valve surgerydagger. Eur J Cardiothorac Surg 2019;55:851-8.

35. Axtell AL, Bhambhani V, Moonsamy P, et al. Surgery is Not Associated with Improved Survival Compared to Medical Therapy in Isolated Severe Tricuspid Regurgitation. J Am Coll Cardiol 2019;74:715-25.

36. Felker GM, Lee KL, Bull DA, et al. Diuretic strategies in patients with acute decompensated heart failure. N Engl J Med 2011;364:797-805.

37. Galie N, Humbert M, Vachiery JL, et al. 2015 ESC/ERS Guidelines for the diagnosis and treatment of pulmonary 
hypertension: The Joint Task Force for the Diagnosis and Treatment of Pulmonary Hypertension of the European Society of Cardiology (ESC) and the European Respiratory Society (ERS): Endorsed by: Association for European Paediatric and Congenital Cardiology (AEPC), International Society for Heart and Lung Transplantation (ISHLT). Eur Heart J 2016;37:67-119.

38. Song JM, Kang DH, Song JK, et al. Outcome of significant functional tricuspid regurgitation after percutaneous mitral valvuloplasty. Am Heart J 2003;145:371-6.

39. Alkhouli M, Berzingi C, Kowatli A, et al. Comparative early outcomes of tricuspid Valve repair versus replacement for secondary tricuspid regurgitation. Open Heart 2018;5:e000878.

40. Kundi H, Popma JJ, Cohen DJ, et al. Prevalence and Outcomes of Isolated Tricuspid Valve Surgery Among Medicare Beneficiaries. Am J Cardiol 2019;123:132-8.

41. De Vega NG. Selective, adjustable and permanent annuloplasty. An original technic for the treatment of tricuspid insufficiency. Rev Esp Cardiol 1972;25:555-6.

42. Navia JL, Nowicki ER, Blackstone EH, et al. Surgical management of secondary tricuspid valve regurgitation: annulus, commissure, or leaflet procedure? J Thorac Cardiovasc Surg 2010;139:1473-82.e5.

43. Wang N, Phan S, Tian DH, et al. Flexible band versus rigid ring annuloplasty for tricuspid regurgitation: a systematic review and meta-analysis. Ann Cardiothorac Surg 2017;6:194-203.

44. Liu P, Qiao WH, Sun FQ, et al. Should a Mechanical or Biological Prosthesis Be Used for a Tricuspid Valve Replacement? A Meta-Analysis. J Card Surg 2016;31:294-302.

45. Choi JW, Jang MJ, Kim KH, et al. Repair versus replacement for the surgical correction of tricuspid regurgitation: a meta-analysis. Eur J Cardiothorac Surg 2018;53:748-55.

46. Perlman G, Praz F, Puri R, et al. Transcatheter Tricuspid Valve Repair With a New Transcatheter Coaptation System for the Treatment of Severe Tricuspid Regurgitation: 1-Year Clinical and Echocardiographic Results. JACC Cardiovasc Interv 2017;10:1994-2003.

47. Orban M, Besler C, Braun D, et al. Six-month outcome after transcatheter edge-to-edge repair of severe tricuspid regurgitation in patients with heart failure. Eur J Heart Fail 2018;20:1055-62.

48. McElhinney DB, Cabalka AK, Aboulhosn JA, et al. Transcatheter Tricuspid Valve-in-Valve Implantation for the Treatment of Dysfunctional Surgical Bioprosthetic
Valves: An International, Multicenter Registry Study. Circulation 2016;133:1582-93.

49. Taramasso M, Hahn RT, Alessandrini H, et al. The International Multicenter TriValve Registry: Which Patients Are Undergoing Transcatheter Tricuspid Repair? JACC Cardiovasc Interv 2017;10:1982-90.

50. Zoghbi WA, Asch FM, Bruce C, et al. Guidelines for the Evaluation of Valvular Regurgitation After Percutaneous Valve Repair or Replacement: A Report from the American Society of Echocardiography Developed in Collaboration with the Society for Cardiovascular Angiography and Interventions, Japanese Society of Echocardiography, and Society for Cardiovascular Magnetic Resonance. J Am Soc Echocardiogr 2019;32:431-75.

51. Utsunomiya H, Itabashi Y, Mihara H, et al. Usefulness of 3D echocardiographic parameters of tricuspid valve morphology to predict residual tricuspid regurgitation after tricuspid annuloplasty. Eur Heart J Cardiovasc Imaging 2017;18:809-17.

52. Prihadi EA, Delgado V, Hahn RT, et al. Imaging Needs in Novel Transcatheter Tricuspid Valve Interventions. JACC Cardiovasc Imaging 2018;11:736-54.

53. Park JB, Kim HK, Jung JH, et al. Prognostic Value of Cardiac MR Imaging for Preoperative Assessment of Patients with Severe Functional Tricuspid Regurgitation. Radiology 2016;280:723-34.

54. Pighi M, Theriault-Lauzier P, Alosaimi H, et al. Fluoroscopic Anatomy of Right-Sided Heart Structures for Transcatheter Interventions. JACC Cardiovasc Interv 2018;11:1614-25.

55. Nashef SA, Roques F, Sharples LD, et al. EuroSCORE II. Eur J Cardiothorac Surg 2012;41:734-44; discussion 744-5.

56. LaPar DJ, Likosky DS, Zhang M, et al. Development of a Risk Prediction Model and Clinical Risk Score for Isolated Tricuspid Valve Surgery. Ann Thorac Surg 2018;106:129-36.

57. Chen Y, Liu YX, Seto WK, et al. Prognostic Value of Hepatorenal Function By Modified Model for End-stage Liver Disease (MELD) Score in Patients Undergoing Tricuspid Annuloplasty. J Am Heart Assoc 2018;7. doi: 10.1161/JAHA.118.009020.

58. Subbotina I, Girdauskas E, Bernhardt AM, et al. Comparison of Outcomes of Tricuspid Valve Surgery in Patients with Reduced and Normal Right Ventricular Function. Thorac Cardiovasc Surg 2017;65:617-25.

59. Sun X, Zhang H, Aike B, et al. Tricuspid annular plane systolic excursion (TAPSE) can predict the outcome of 
isolated tricuspid valve surgery in patients with previous cardiac surgery? J Thorac Dis 2016;8:369-74.

60. Kim JB, Jung SH, Choo SJ, et al. Surgical outcomes of severe tricuspid regurgitation: predictors of adverse clinical outcomes. Heart 2013;99:181-7.

61. Anand A, Harley C, Visvanathan A, et al. The relationship between preoperative frailty and outcomes following transcatheter aortic valve implantation: a systematic review and meta-analysis. Eur Heart J Qual Care Clin Outcomes 2017;3:123-32.

62. Min SY, Song JM, Kim JH, et al. Geometric changes after tricuspid annuloplasty and predictors of residual tricuspid regurgitation: a real-time three-dimensional

Cite this article as: Wang TKM, Unai S, Xu B. Contemporary review in the multi-modality imaging evaluation and management of tricuspid regurgitation. Cardiovasc Diagn Ther 2021;11(3):804-817. doi: 10.21037/cdt.2020.01.06 echocardiography study. Eur Heart J 2010;31:2871-80.

63. Onoda K, Yasuda F, Takao M, et al. Long-term follow-up after Carpentier-Edwards ring annuloplasty for tricuspid regurgitation. Ann Thorac Surg 2000;70:796-9.

64. Sorabella RA, Mamuyac E, Yerebakan H, et al. Residual Tricuspid Regurgitation following Tricuspid Valve Repair during Concomitant Valve Surgery Worsens Late Survival. Heart Surg Forum 2015;18:E226-31.

65. Taramasso M, Gavazzoni M, Pozzoli A, et al. Tricuspid Regurgitation: Predicting the Need for Intervention, Procedural Success, and Recurrence of Disease. JACC Cardiovasc Imaging 2019;12:605-21. 